\title{
A New Method Based on Field Strength for Road Infrastructure Risk Assessment
}

\author{
Yi Li $\mathbb{D}$ and Yuren Chen \\ The Key Laboratory of Road and Traffic Engineering, Ministry of Education, College of Transportation Engineering, \\ Tongji University, No. 4800 Cao'an Hwy., Shanghai 201804, China \\ Correspondence should be addressed to Yi Li; smilesusanly@163.com
}

Received 23 April 2018; Revised 3 July 2018; Accepted 6 September 2018; Published 25 September 2018

Guest Editor: Xiaobo Qu

Copyright (C) $2018 \mathrm{Yi} \mathrm{Li} \mathrm{and} \mathrm{Yuren} \mathrm{Chen.} \mathrm{This} \mathrm{is} \mathrm{an} \mathrm{open} \mathrm{access} \mathrm{article} \mathrm{distributed} \mathrm{under} \mathrm{the} \mathrm{Creative} \mathrm{Commons} \mathrm{Attribution} \mathrm{License,}$ which permits unrestricted use, distribution, and reproduction in any medium, provided the original work is properly cited.

\begin{abstract}
Because road infrastructures have significant impact on driving safety, their risk levels need to be evaluated dynamically according to drivers' perception. To achieve this, this paper proposes two field strength models to quantify the impact of road infrastructures on drivers. First, road infrastructures are classified into two types (continuous and discrete). Then, two field strength models for these types are proposed. Continuous field strength model describes the impact of long-belt-shape infrastructure by differential and integral methods. Discrete field strength model describes the static and dynamic characteristics of infrastructures. This model includes four parameters: mass of vehicles, mass of infrastructures, warning level, and kinetic energy of road infrastructures. The field strength is a relative concept, which changes with vehicle state. At the end of this paper, risk assessment principles are listed to clarify the nature of road infrastructure risk evaluation. A workflow of risk assessment and a case study are presented to illustrate the application of this novel method. The result of this study shows that (1) the field strength is positively related to its risk level; (2) the distribution of road infrastructure risks explains driver behaviour correctly; (3) drivers tend to keep driving in low-risk area. These findings help to explain the impact mechanism of road infrastructures on drivers, which can be applied in AI-based driving assistance system in the future.
\end{abstract}

\section{Introduction}

Road infrastructures are basic components of traffic environment. China has built more than $40,000 \mathrm{~km}$ highway in the last five years. In such circumstance, a large number of roadside facilities need to be assessed. They are not only the structure of road alignment, but also the guidance for drivers. Past studies have proposed a lot of models and systems for road infrastructure assessment.

(1) Studies on the Relationship between Road Infrastructure and Driver's Visual Perception. Drivers do not capture infrastructure information through direct contact. More than $80 \%$ of such information is obtained from visual perception $[1,2]$. Plenty of experiments have been done to verify it. It showed that different traffic environment would result in different driving intention and behaviour [3]. Road line ratio from driver's view was collected through field tests by Victor [4]. The results showed that it would decrease with the climb of driver's vision pressure. Wang et al. (2006) regarded the road infrastructure information as a series of stimulation. If the stimulation density from the infrastructure was in a proper range, then driver behaviour would be safe. On the other hand, some eye movement criteria (spot distribution, spot strength, etc.) were used to evaluate the visual burden caused by small radius and small angle of road alignment [5]. The results indicated that sharp radius would result in large visual burden for drivers. Different sweeping duration on road condition also showed some certain impact on lane keeping behaviour [6].

Other researchers focused on identifying such influence at intersections where the infrastructures were more various. Corresponding models were built to describe the relationship between real-world experimental data and driver's inner pressure [7]. Werneke and Vollrath [8] did field and simulated tests at intersections, and they concluded that poor planning of infrastructures near intersections would lead to severe vehicle collisions. Similar tests were conducted on mountain highway. The cross analysis between driver's vision pressure and psychological tension confirmed the importance of 
proper road shoulder width and access management [9-11]. Besides, drivers' speed adaption behaviour was proved to vary with different road infrastructures and traffic complexity conditions. Complex infrastructure information would significantly result in frequent speed adaption behaviour [12].

Based on such tests and analysis, it can be seen that scientific infrastructure arrangement and proper infrastructure information are important for driving safety $[13,14]$.

(1) Studies on Driver's Vision Pressure Field. As a part of "Human-Vehicle-Road" circle, road infrastructures will influence the whole driving environment. Therefore, the risk of road infrastructure should be assessed dynamically from the view of its user-driver. The risk assessment is only valid when the interaction between infrastructure and driver does exist. Based on such understanding, driver's vision pressure field theory is proposed as the extension of artificial potential field theory [15]. In his theory, there was a potential field in which objects would attract and exclude the moving objects, and this was the source of movement. This theory assumed that objects were pushed under virtual force. It has been widely applied in solving robot path planning problem. Similarly, the moving vehicles can be regarded as a kind of movement in a potential field composed of road infrastructures. Then, many pressure field models were built according to this theory: (1) discrete pressure field model [16] explained driver's decision pattern; (2) vehicle path planning model combined pressure field theory and elastic band theory to predict vehicle's path (Thomas and Thorsten 2008); (3) driver's workload theory helped to estimate the impact of other traffic on drivers [17]. With the development of road scanning technology, more infrastructure information and indexes are available for precise analysis [18]. Moreover, the pressure field was simulated to visualize its impact on vehicle movement [19]. These studies helped to quantify the impact of road infrastructures on drivers, and such impact was also known as driving safety field proposed by Wang et al. $[20,21]$. In driving safety field, road infrastructures were the sources of static potential field that had an important role in collision warning algorithm. Based on this, a dynamic 3D virtual hazard potential field model was built to calculate the field energy of infrastructures for driving decision assistance $[22,23]$. It can be seen that the whole road environment is a physical field, and the road infrastructures build the framework of it (Ni 2013).

Past studies mainly focus on four aspects: (1) the relationship between pressure field and real road environment; (2) what is the physical rule of this pressure field; (3) how to explain such field phenomenon from drivers' view; (4) how to apply this theory in road risk assessment. Therefore, driver's vision pressure field still needs a deep study on the interaction between road infrastructures and drivers. This paper will focus on its application on risk assessment of road infrastructure.

(3) Studies on Road Infrastructure Risk Assessment Methods. Road infrastructure assessment is a major topic in traffic safety. In India, planning and building safe road infrastructure have been proposed as a high priority [24].
To reduce the negative influence of road infrastructures, the project MARVin in Austria built a database which included accident data and road parameters (radius, gradient, etc.). The risk of road infrastructure was assessed through comparison between tested road and nonaccident road. Two roads shared similar parameters, but they had different speed limits or weather conditions. Then high-crash-risk road sections or infrastructures can be spotted [25]. A similar method was used in risk model proposed by Appleton [26]. By comparison with benchmark road in New Zealand, the researcher got personal risk and collective risk scores. The score was the product of risk level and feature extent. Zhang and $\mathrm{Hu}$ [27] built a modified Bayesian network model to assess the facility risks on freeways, in which traffic data or field survey data were applied to calibrate parameters of the model. The results showed that smaller model result resulted in higher facility risk, and the threshold was 0.5. Bayesian network was also applied in multirisk assessment of road infrastructure system, and fully probabilistic approach was adopted to integrate multirisk interactions at both hazard and fragility levels [28].

Road infrastructure assessment is also a part of macroscopic road management, which focuses on road-user risk caused by road infrastructure. CEDR-project developed a risk assessment framework to obtain the risk of pavement, structure, and drainage in network level for most of the European road administrations [29]. Specifically, systematic risk model described the relationship among road infrastructure, vehicle, and driver. Multidimensional probability distribution quantified cause-and-effect chain of the risk impact of infrastructure on vehicle [30]. Other studies took the concept of infrastructure risk as collision risk. No collision means no risk [31]. Besides, improper facility was also a main reason for unsafe behaviour, such as speeding [32], distraction [33], and run-off-road crashes [34, 35].

Recently, intelligent vehicles which can collect vehicle movement and roadside information have been widely developed. With such technology, a series of experiments were conducted in northern Virginia. The results indicated that wide shoulder had significant impact on driver behaviour change [36]. Severe accidents would increase with improper slopes, bends, or pavement surface conditions for $\mathrm{HGV}$ (Heavy Goods Vehicles) [37].

It can be seen that past studies on risk assessment of road infrastructure mainly focused on performance comparison and systematic assessment. They presented general comments, but they ignored the interaction between infrastructures and users (e.g., drivers) [38]. Recently, Scott-Parker et al. [39] have tested drivers stress in response to different infrastructures, but they did not explain its impact mechanism. To overcome this weakness, this paper regards drivers as assessors of road infrastructures. Their subjective visual pressure is the key to evaluate the risk levels of nearby facilities. Corresponding field strength models and risk assessment methods are discussed in detail in this paper. This helps to quantify the relationship between driver behaviour and road infrastructures, and it also presents a better understanding on the nature of road infrastructure risk assessment. The concept 

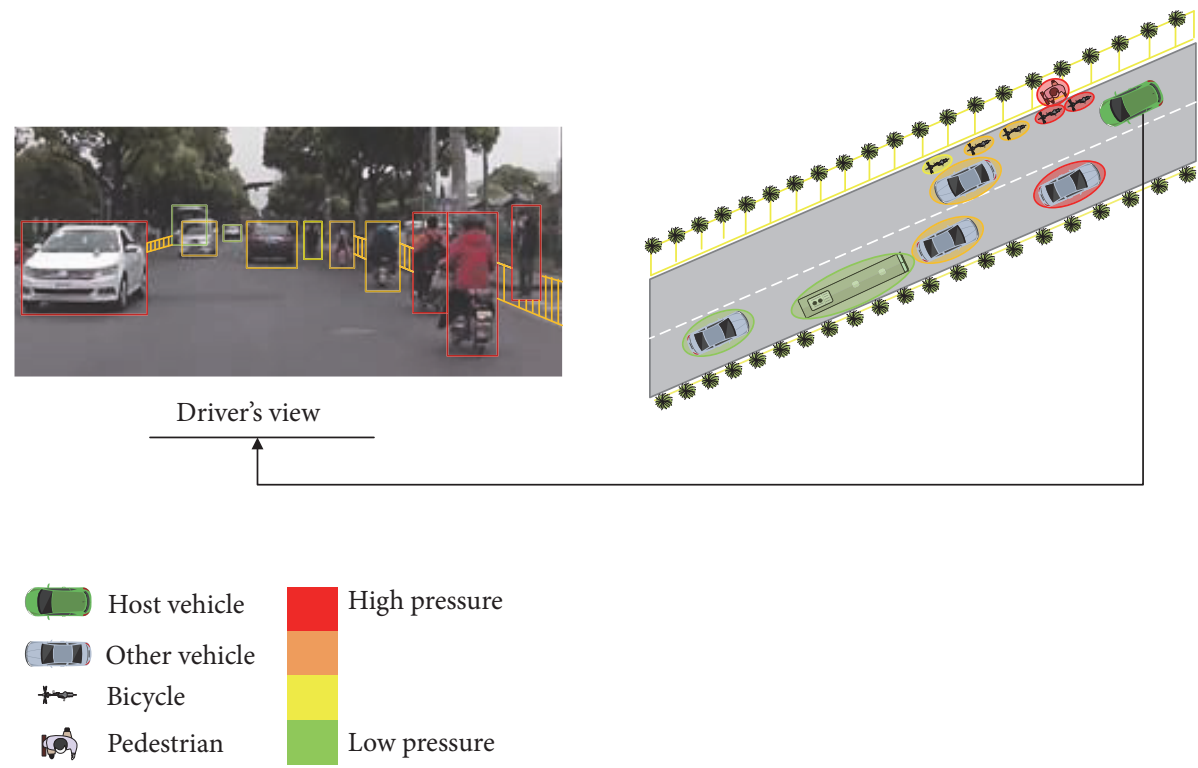

FIgURE 1: Conceptual image of pressure field from driver's view.

of pressure sources and field strength models may become the foundation of risk assessment system.

This paper is organized as follows. Section 1 introduces the achievements and weaknesses of past studies on driver's vision pressure field and road infrastructure risk assessment methods. Section 2 classifies some common road infrastructures based on pressure sources. Section 3 describes the field strength model of two road infrastructure types. Section 4 presents some assessment principles and a case study. Section 5 concludes the achievements and limits of this paper.

\section{Classification of Road Infrastructure Based on Pressure Sources}

In a complex traffic system, road infrastructure is a critical part. It does not have direct contact with vehicles, but it has influence on driver behaviour. In this paper, we define this mechanism as pressure field, which is not visible but measurable. Figure 1 shows a conceptual image of pressure field from driver's view.

The chance of direct collision between vehicle and road infrastructure is small; however driver behaviour continuously changes under the restrictions of nearby road infrastructures (including road geometry, road facilities, and other road users). This means that the pressure field is similar to gravity field, electric field, and magnetic field, which can force the vehicles to accelerate, decelerate, or change lane. If the risk level of road infrastructure is too high, it will form a stressful road environment for drivers, which may lead to unsafe driver behaviour. Therefore, a scientific risk assessment method for road infrastructure is necessary to understand and quantify such impact.

As the basis of road infrastructure assessment, we first need to classify some common road infrastructures. The classification is based on physical features of road infrastructures.
Although other factors like weather, visibility, time of day, etc. may also have some impact on driver behaviour [40, 41], we eliminate these factors by experiments only on sunny days during 8:00 to 15:00 with good visibility in this research.

There are various shapes of infrastructures on road, but most of them are continuous type and discrete type. Continuous road infrastructures have continuous impact on the vehicles during the driving process. They are mostly longbelt-shape facilities standing or attaching to the road surface. Discrete road infrastructures are arranged separately on road. Although there are many kinds of discrete infrastructures, they can be mainly divided into two types: independent and centralized. Typical independent discrete infrastructures are traffic signs or signals. Centralized discrete infrastructures consist of a number of independent discrete infrastructures, such as disperse barriers. Tables 1 and 2 show some typical continuous and discrete road infrastructures, respectively.

The road infrastructures have two kinds of effect on vehicles: exclusion and attraction. The exclusion effect forces the vehicles to keep a certain distance from pressure sources. The attraction effect, however, leads the vehicles to move towards the pressure sources. Table 3 shows the pressure effect of some typical pressure sources.

Some elements in Table 3 are listed in both exclusion and attraction columns because they show different impacts on different vehicle types. For example, bus lane has attraction effect on buses in rush hour. However, it shows exclusive effect on other cars. Besides, in off-peak hours it becomes an ordinary lane, and it has no restriction at all. Meanwhile, some elements only have attraction effect on some vehicles in some specific circumstances. For example, emergency parking lane has no effect on normal vehicles, but it has great attraction effect on out-of-control vehicles.

Based on the above classification and the discussion, we will build two models to quantify the impact of these two road infrastructure types on drivers. 


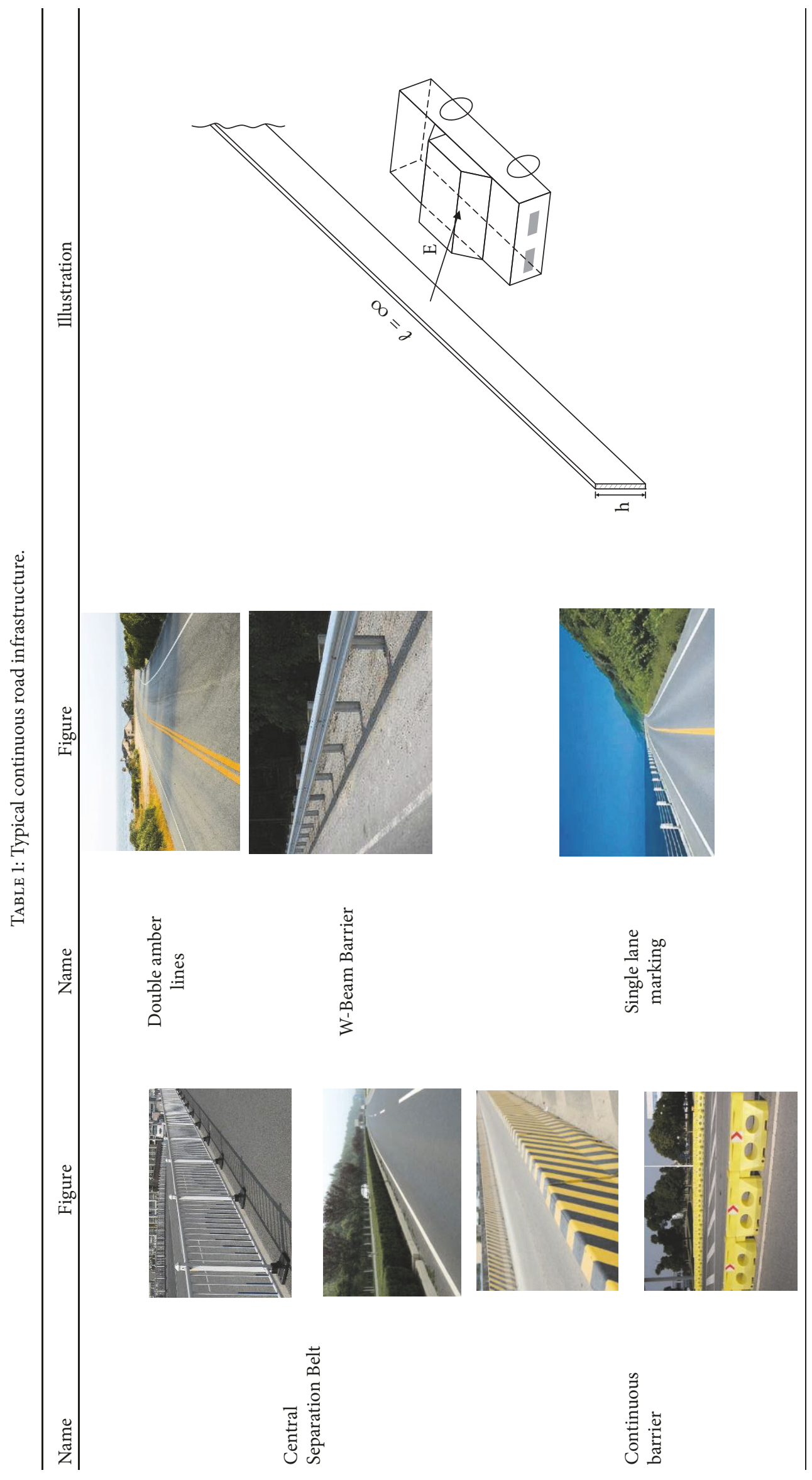




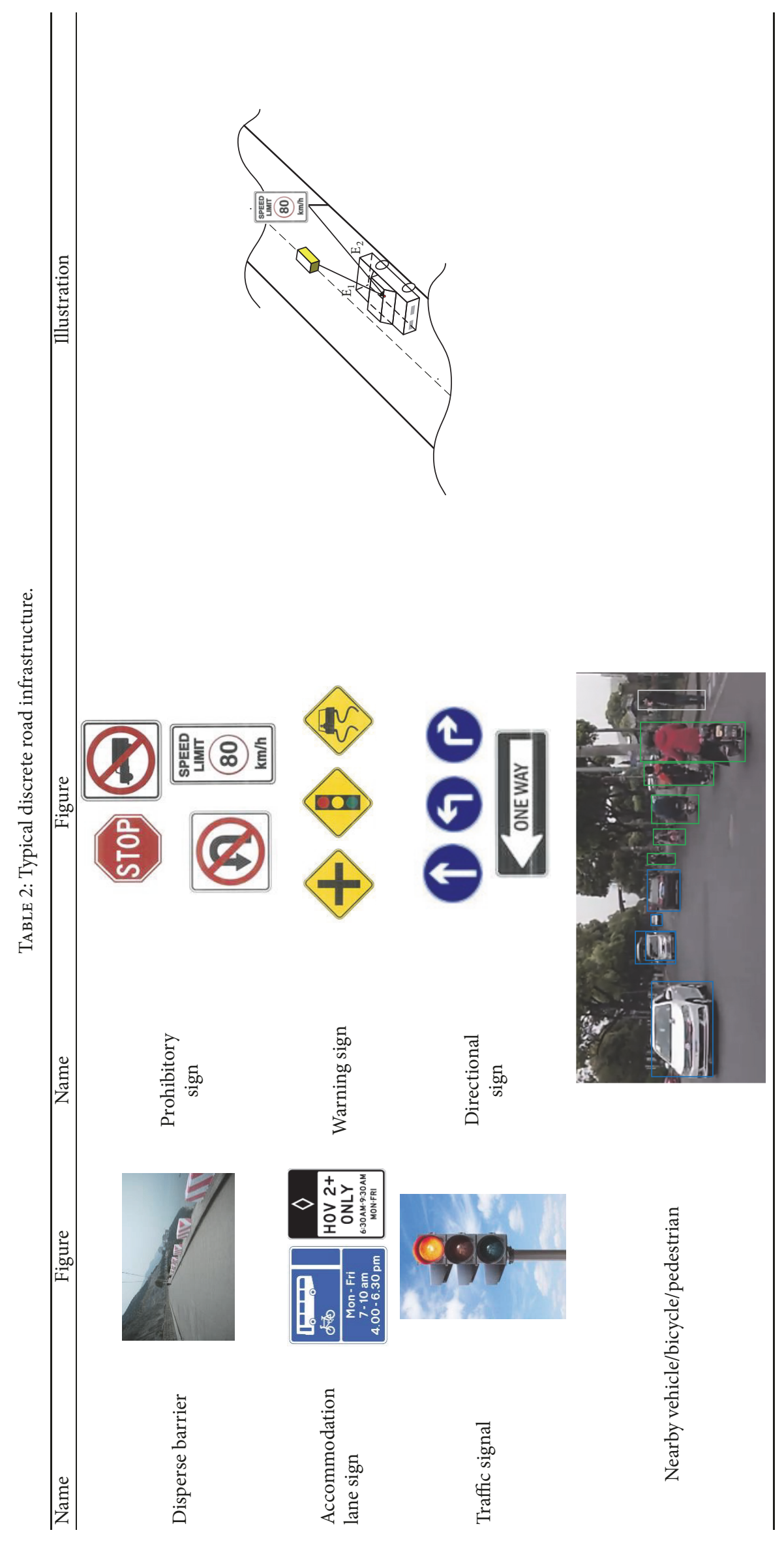


TABLE 3: The pressure effect of typical pressure sources.

\begin{tabular}{|c|c|c|}
\hline \multirow{2}{*}{ Pressure sources } & \multicolumn{2}{|c|}{ Pressure effect } \\
\hline & Exclusion & Attraction \\
\hline \multirow{2}{*}{ Lane marking } & Prohibitory marking & Amber dash marking ${ }^{\mathrm{b}}$ \\
\hline & Warning marking & Lane dash marking ${ }^{\mathrm{b}}$ \\
\hline \multirow{5}{*}{ Road sign } & Prohibitory sign & Directional sign \\
\hline & Warning sign & Intersection waiting area ${ }^{\mathrm{b}}$ \\
\hline & Accommodation lane sign ${ }^{a}$ & Accommodation lane sign \\
\hline & Intersection guide line $\mathrm{a}^{\mathrm{a}}$ & Intersection guide line $\mathrm{e}^{\mathrm{a}}$ \\
\hline & Traffic signal (red/yellow) & Traffic signal (green) \\
\hline \multirow{3}{*}{ Barrier } & Guardrail & Escape lane $^{\mathrm{b}}$ \\
\hline & Crash barrier & Emergency parking lane $\mathrm{b}^{\mathrm{b}}$ \\
\hline & Working zone & \\
\hline
\end{tabular}

Note. a. The element shows different pressure effect under different circumstances; b. the element only is shown as a certain kind of pressure effect in some circumstances.

\section{Field Strength Model of Different Road Infrastructure Types}

3.1. Continuous Road Infrastructure Field Strength Model. To simplify the calculation, the host vehicles is represented by a rectangle object $(a \times b \times c)$. The origin of the coordinate system is the geometrical center. The positive Y-axis points to the direction of vehicle movement. Figure 2 shows the simplified vehicle model and coordinate system.

Because a continuous road infrastructure always has a relative long dimension compared to other dimensions (see Figure 2), the differential analysis can be used to select the microsource unit of such item ( $d s$ : microunit, width $=d z$, length $=\infty$ ). Figure 3 shows the impact of field strength of microsource unit on vehicle in $z$ plane.

Then, the corresponding field strength of this microsource unit can be calculated as follows.

$$
d E=\frac{\vartheta}{\alpha \cdot l} d z=\frac{\tau}{\alpha \cdot \pi \cdot\|\vec{x}+\vec{y}+\vec{z}\|_{2}} d z
$$

where $d E$ is the field strength of microsource unit $d s$; $d z$ is the width of microsource unit $d s ; \alpha$ is the reliability parameter of continuous field source, which consists of four levels: 1-rigid, 2-semirigid, 3-flexible, and 4-traversable.

$l$ is the boundary length of field range. When the infrastructure is in the center of road, the field range is centrosymmetric distributed around pressure sources. When it locates at roadside, the range covers a 1/4 circle area around pressure sources.

$\tau$ is the characteristic parameter of continuous field source, which shows the protection intensity of field source. The value of this parameter depends on the "Specification for Design of Highway Safety Facilities" (JTG D81-2017) in China. To cover different levels of guardrail and lane marking, $\tau$ is divided into eight levels, shown in Table 4 .

According to Figures 3 and 4, the microfield source is infinitely small along $z$-axis, so the vehicle is parallel to the microfield source along $y$-axis. If the infrastructure locates

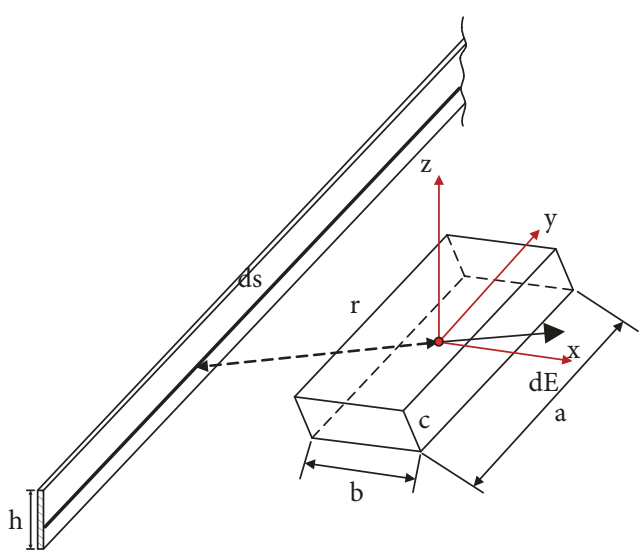

Figure 2: Simplified vehicle and coordinate system.

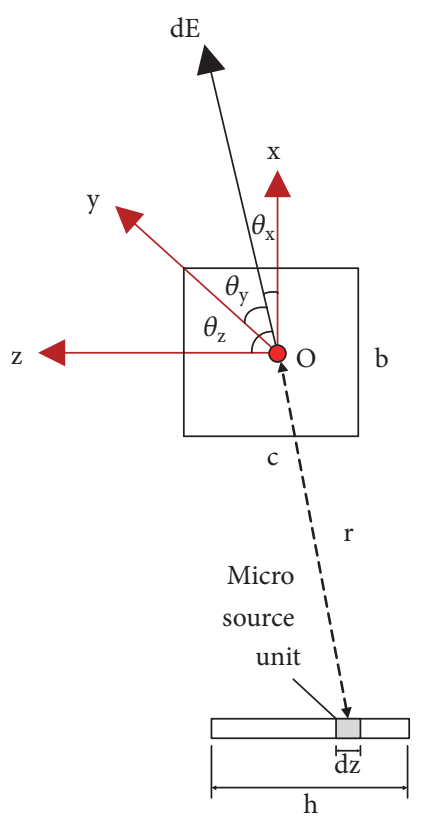

$d E$ : the field strength of micro source unit $d s$

FIGURE 3: Field strength of microsource unit. 


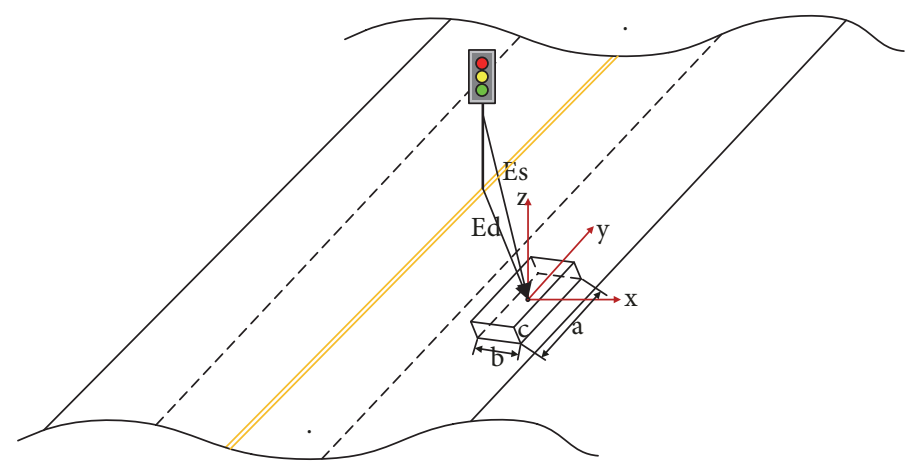

FIGURE 4: Static field strength (Es) and dynamic field strength (Ed) of independent pressure source.

TABLE 4: The level of characteristic parameter of continuous field source.

\begin{tabular}{llcl}
\hline Protection intensity & $\tau$ & Protection intensity & $\tau$ \\
\hline Dash lane marking & 1 & $\mathrm{~A} / \mathrm{A}_{\mathrm{m}}$-class protection & 5 \\
White lane marking & 2 & $\mathrm{SB} / \mathrm{SB}_{\mathrm{m}}$-class protection & 6 \\
Amber lane marking & 3 & $\mathrm{SA} / \mathrm{SA}_{\mathrm{m}}$-class protection & 7 \\
B-class protection & 4 & $\mathrm{SS}$-class protection & 8 \\
\hline
\end{tabular}

in the center of road, the field strength along $x$-axis can be calculated as follows.

$$
\begin{aligned}
d E_{x} & =\frac{\tau \cdot d z}{\alpha \cdot l} \cdot \cos \theta_{x} \\
& =\frac{\tau \cdot x / \sqrt{x^{2}+z^{2}}}{\alpha \cdot \pi \cdot \sqrt{(|\mathrm{x}|-b / 2)^{2}+(|z|-b / 2 \cdot z / x)^{2}}} d z \\
& =\frac{\tau}{\alpha \pi} \cdot \frac{|\mathrm{x}|-b / 2}{(|\mathrm{x}|-b / 2)^{2}+(|z|-b / 2 \cdot z / x)^{2}} d z
\end{aligned}
$$
follows.

Then the whole field strength function can be obtained as

$$
\mathrm{E}_{x}=\int_{-c / 2}^{h-c / 2} \frac{\tau}{\alpha \pi} \cdot \frac{|\mathrm{x}|-b / 2}{(|\mathrm{x}|-b / 2)^{2}+(|z|-b / 2 \cdot z / x)^{2}} d z
$$

This function shows that the field strength along $x$ axis will change with the distance between the vehicle and infrastructure.

3.2. Discrete Road Infrastructure Field Strength Model. Compared with continuous infrastructures, discrete infrastructures not only appear occasionally but also have instruction and guidance information. The field strength of a discrete infrastructure consists of two parts: static field strength and dynamic field strength (see Figures 4 and 5).

The overall field strength can be obtained as follows.

$$
\overrightarrow{E_{d i s}}=\overrightarrow{E_{s}}+\overrightarrow{E_{d}}
$$

where $\overrightarrow{E_{d i s}}$ is the overall field strength of a discrete infrastructure pressure source; $\overrightarrow{E_{s}}$ is the static field strength of a discrete infrastructure pressure source; $\overrightarrow{E_{d}}$ is the dynamic field strength of a discrete infrastructure pressure source.

The concept of static field strength is similar to the field strength of continuous sources. It reflects the inherent characteristics of an independent pressure source. The distribution of its field intensity is within the circle (in the middle of road) or semicircle (on the side of road) around the pressure sources. Figure 6 shows the decomposition of static field strength.

In Figure 6, the mass center is the original point of field strength. Its coordinate is as follows.

$$
\begin{aligned}
& \bar{x}=\frac{\iiint x u(x, y, z) d v}{m}=\frac{\iiint x d v}{V} \\
& \bar{y}=\frac{\iiint y u(x, y, z) d v}{m}=\frac{\iiint y d v}{V} \\
& \bar{z}=\frac{\iiint z u(x, y, z) d v}{m}=\frac{\iiint z d v}{V}
\end{aligned}
$$

where $(\bar{x}, \bar{y}, \bar{z})$ is the coordinate of mass center; $V$ is the volume of field source; $m$ is the mass of field source.

The distance $\left(r_{1}\right)$ between the field source and vehicle is as follows.

$$
\mathrm{r}_{1}=\sqrt{\left(\sqrt{\bar{x}^{2}+\bar{y}^{2}}+\frac{b}{2 \cos \theta_{x}}\right)^{2}+\left(|\bar{z}|+\frac{b \cot \theta_{z}}{2 \cos \theta_{x}}\right)^{2}}
$$

Then the static field strength can be obtained:

$$
\mathrm{E}_{s}= \begin{cases}\frac{\beta \cdot m_{\text {source }}}{r_{1}^{2}}, & \text { independent pressure source } \\ \sum_{i=1}^{n} \frac{\beta_{i} \cdot m_{i}}{r_{1}^{2}}, & \text { centralized pressure source }\end{cases}
$$

where $m_{\text {souce }}$ is the inertial characteristics of field source, which can be quantified by mass; $\beta$ is the warning level of field source, which is divided into three levels; see Table 5.

Compared with static field strength, dynamic field strength shows guidance effect on traffic flow, so it covers only a specific direction of traffic flow. In reference to pointcharge field theory in physics, discrete pressure source and 


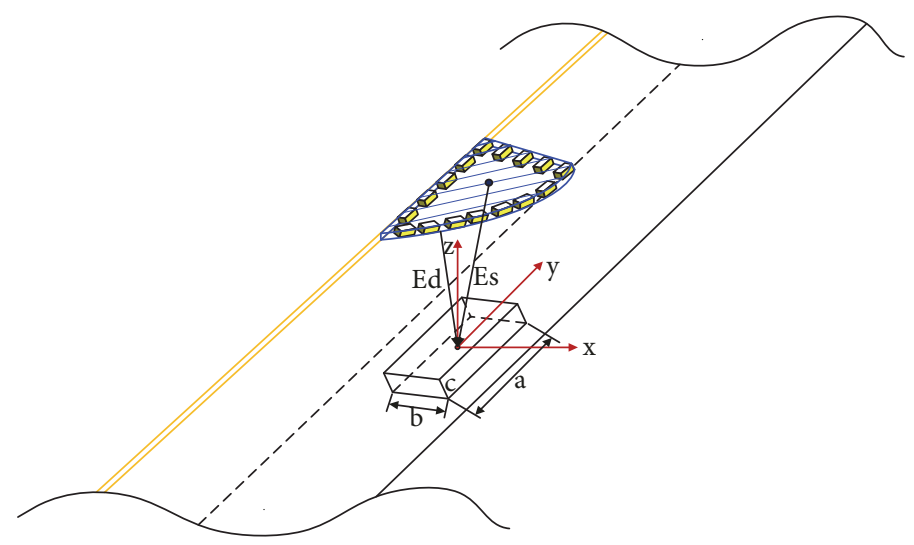

FIGURE 5: Static field strength (Es) and dynamic field strength (Ed) of centralized pressure source.

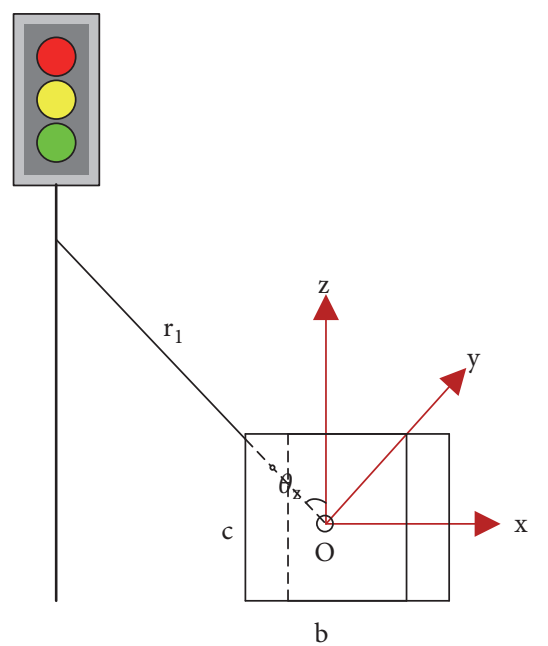

(a)

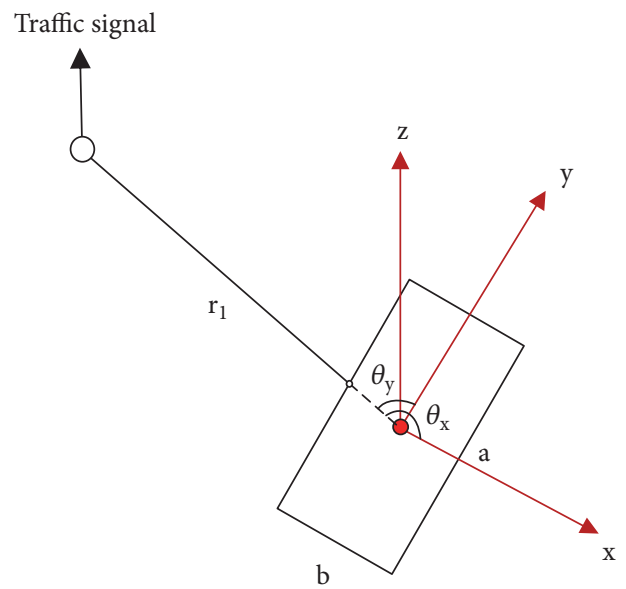

(b)

FIGURE 6: Static field strength decomposition. ((a) longitudinal, (b) overlook).

TABLE 5: Warning level of field source.

\begin{tabular}{|c|c|}
\hline Level & Infrastructure \\
\hline 1 & $\begin{array}{l}\text { Warning sign/Accommodation lane sign/Directional } \\
\text { sign }\end{array}$ \\
\hline 2 & Prohibitory sign/Traffic signal \\
\hline 3 & Disperse barrier \\
\hline
\end{tabular}

vehicle can be regarded as a couple of energetic bodies, so the dynamic field strength is as follows.

$$
\mathrm{E}_{d}= \begin{cases}\frac{\beta \cdot \zeta}{r_{2}{ }^{2}}, & \text { independent pressure source } \\ \frac{\beta_{c e n \cdot \zeta}}{r_{2}{ }^{2}}, & \text { centralized pressure source }\end{cases}
$$

where $r_{2}$ is the vertical distance from vehicle to infrastructure, $r_{2}=\sqrt{x^{2}+y^{2}} ; \beta_{c e n}$ is the warning level of the group field source; $\zeta$ is the warning kinetic energy of field source, which is the required kinetic energy for host vehicle. For example, in car-following situation $\zeta=(1 / 2) m_{\text {front car }} v_{\text {front car }}^{2}$; in speed limit situation $\zeta=(1 / 2) m_{\text {host }} v_{\text {limit }}^{2}$.

\section{Risk Assessment Principles and Case Study}

In this section, the above models are utilized to assess the risk of road infrastructures with a case study.

First, some risk assessment principles need to be declared. (In this paper, risk source is equal to pressure source.)

(1) Variability of Risk Source Type under Different Scenes. In Section 2, we classify the road infrastructures into two types. In the case of multisource combination, the types of some field sources change with their directional text and time limit. This leads to an opposite risk assessment result (see the example of bus lane in Section 2). Therefore, before assessing the road infrastructure risk, we should check the pressure source type and state first. 
TABLE 6: Indexes of pressure sources.

\begin{tabular}{|c|c|c|c|c|}
\hline No & Infrastructure & Pressure effect & Indexes & Illustration \\
\hline 1 & $\begin{array}{l}\text { Continuous } \\
\text { barrier }\end{array}$ & Exclusion & $\begin{array}{c}\tau=5 \\
\alpha=2 \\
\mathrm{~h}=2.5 \mathrm{~m}\end{array}$ & \\
\hline 2 & Lane marking & Attraction & Dash line & \\
\hline 3 & $\begin{array}{l}\text { Discrete } \\
\text { barrier }\end{array}$ & Exclusion & $\begin{array}{c}m_{\text {source }}=225 \mathrm{~kg} \\
\mathrm{~h}=0.7 \mathrm{~m}\end{array}$ & \\
\hline 4 & Host vehicle & I & $\begin{array}{c}m_{\text {host }}=1500 \mathrm{~kg} \\
\mathrm{~b}=1.8 \mathrm{~m} \\
\mathrm{c}=2 \mathrm{~m} \\
v_{\text {host }}=40 \mathrm{~km} / \mathrm{h}\end{array}$ & \\
\hline * & Lane width & l & $3.5 \mathrm{~m}$ & \\
\hline
\end{tabular}

(2) Traffic-Rule-Based Pressure Source Priority. Traffic rules and guidance clarify the right-of-way and drivers' behaviour standard. Similarly, we divide the pressure source priority into four levels. Among these priority levels, driver's safety and vehicle collision prevention are the most important. The specific priority levels are described as follows.

Priority 1: Fixed road infrastructure in driver's view.

Priority 2: Prohibitory sign/line or signal.

Priority 3: Warning sign/line or signal.

Priority 4: Traversable line, directional sign/line or signal.

In the process of pressure combination, the priority of each pressure source needs to be evaluated. First, combine the field strength of Priority 1 . Then, add the combination results of lower priorities on the previous result. This forms a priority-based level system, which is easy for intelligent detection and analysis in the future.

(3) The Relativity of Road Infrastructure Risk. There are various risk sources (pressure sources) in road environment and different drivers would perceive different risk levels in multisource field. Generally, such characteristic can be explained in two aspects.

(1) When facing with the same pressure source, drivers will perceive different priority according to their vehicle types. For example, some road infrastructures only restrict the passage of truck, so they have high priority for truck drivers and low priority for vehicle drivers.

(2) When the same driver drives the same vehicle along the road several times, the risk level and combination of road infrastructures vary with vehicle position and vehicle state.

Therefore, the road infrastructure risk is a changeable and relative factor. It needs to be analyzed and calculated specifically in each scenario.

Next, according to the principles discussed above, we put forward an example (a section on Cao'an Hwy., Shanghai, China) with continuous and discrete road infrastructures to show the workflow of risk assessment based on the models proposed above. The indexes of pressure sources are listed in Table 6.

To explain the risk assessment clearly, we draw the assessment workflow with the example data in Figure 7. Each view and vehicle state of current moment during driving process is a series of data input for the assessment. (i) Firstly, each infrastructure in driver's view is classified according to its physical features (continuous or discrete), and its pressure indexes are also collected with vehicle state data. (In this paper, we assume that all indexes of road infrastructures are digitalized and stored in a database, which can be collected intelligently on road.)

(ii) Secondly, based on the vehicle and pressure source data, we can grade the priority level (e.g., P1 and P4) of each infrastructure.

(iii) Thirdly, the field strength of each priority is obtained based on the above results of classification and individual field strength calculation (P1: 11.32 175.68, P4: -14.14 -4.46). Moreover, the overall field strength of the whole road infrastructure environment in driver's view can be obtained (E: -2.15 171.22).

(iv) Then, the risk of each infrastructure needs to be assessed according to a risk level guide which can be customized to meet road authorities' needs. In this particular example, four risk levels are provided in Table 7. If the risk level of infrastructure is below the minimum threshold, then the infrastructure is regarded as a safe one. If the field strength is within the other three intervals, corresponding risk level can be calibrated.

(v) Finally, we can get the risk assessment result of current view of road infrastructure.

In this example, the discrete barrier on roadside shows high risk for drivers on the nearest lane. Continuous barriers are safe for driving, and the dash lane markings have attractive effect on vehicle movement.

To show the impact of risk assessment results on driver behaviour, corresponding vehicle trajectories (pink dots and white arrow) are drawn on the field strength diagram. Figure 8 shows the test data of 20 drivers and average vehicle trajectory in this situation. It can be seen that all drivers chose to change lane in order to keep away from the high field strength area. They always kept their trajectories in low/no risk area. However, these trajectories were not the same because each driver had his/her own vision perception and risk assessment result. If some drivers got high pressure 


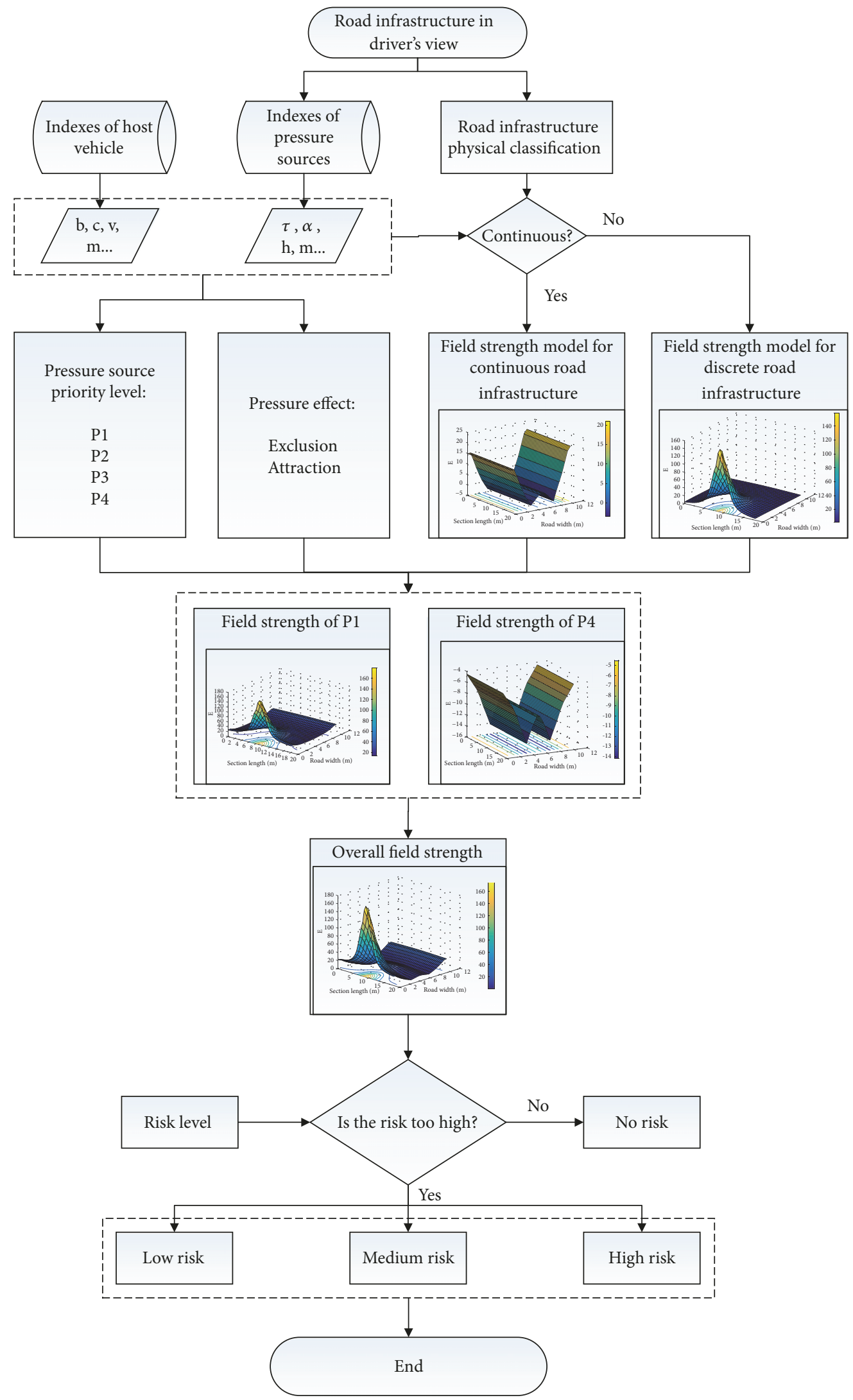

FIGURE 7: Workflow of road infrastructure risk assessment with test data. 


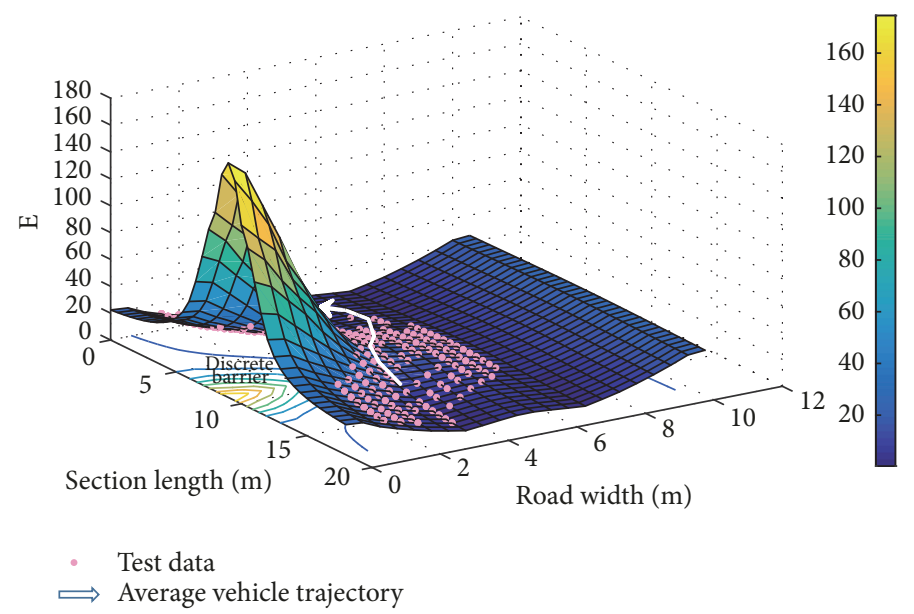

FIGURE 8: Vehicle trajectories in 3D field strength diagram.

TABLE 7: Risk levels of road infrastructure (case study).

\begin{tabular}{lcc}
\hline Risk level & Field strength & Explanation \\
\hline No risk & $\mathrm{E}<60$ & The infrastructure is good enough for safe driving. \\
\hline Low risk & $60 \leqslant \mathrm{E}<100$ & $\begin{array}{c}\text { The infrastructure is safe for most drivers, but it is risky for a small part of } \\
\text { drivers in harsh weather. }\end{array}$ \\
\hline $\begin{array}{l}\text { Medium risk } \\
\text { High risk }\end{array}$ & $100 \leqslant \mathrm{E}<140$ & $\begin{array}{c}\text { The infrastructure is quite safe under normal condition, but it is risky for a } \\
\text { large part of drivers in harsh weather. }\end{array}$ \\
\hline
\end{tabular}

earlier than others, then their psychology index (such as heart rate) would force them to react more quickly [42]. Although drivers' reactions are different, the average behaviour shows that (1) the field strength model can quantify the road infrastructure risk correctly; (2) driver behaviour will change with the risk levels of nearby road infrastructures, so it verifies the necessary of this assessment study and the importance of road infrastructure planning.

\section{Conclusion}

This paper presents a new method and novel field strength models for road infrastructure risk assessment. Past risk assessment methods are mainly based on the physical indexes of road infrastructures. Driver's perception and its impact mechanism are neglected in such methods, so they are static and inflexible. The risk assessment method we present here takes driver's subjective visual perception of road infrastructures as an important factor. This driver-vision-based method helps to quantify such process, which overcomes the weakness of traditional methods. In other words, our method provides a dynamic and specific way to measure the risk impact of road infrastructures on driver behaviour. The main contributions of this research are as follows. The classification method of typical road infrastructures is provided. All infrastructures are components of road environment, which can be quantified by field strength. Because different kinds of road infrastructures have different pressure impact on drivers, we build two field strength models. Continuous field strength model describes the persistent impact on driving behaviour. Discrete field strength model shows the static and dynamic impact of the infrastructures on drivers. Based on the above models and analysis, three risk assessment principles show the nature of risk source evaluation. Finally, the workflow of risk assessment is presented with a case study, and corresponding risk levels are listed and explained.

However, there are still some limits in this research. Firstly, we assume that all characteristics of road infrastructures have been intelligently collected. This may be possible for some advanced autopilot vehicles or connected vehicles, but it is hard for traditional vehicles or platforms. Secondly, as each frame of recorded videos from drivers' view needs to be analyzed, the amount of calculation is huge. Therefore, highspeed calculation, transmission, and distributed procession will be thresholds for online analysis. Thirdly, we only discuss the overall risk level of road infrastructures for all drivers. Nevertheless, this risk assessment method is based on the field strength captured from driver's view, so different drivers may have different driving preference and decisions during the trip, which may lead to some deviation among drivers. Fourthly, we mainly divide common road infrastructures into continuous type and discrete type, but there are still various infrastructures and features to be analyzed for precise assessment in the future. Besides, the influence of bad weather and poor visibility is not considered in the pressure calculation in this research, which needs to be analyzed with more 
test data under various weather conditions. In the following studies, a more elaborate and dynamic risk evaluation system is necessary for personalized service.

\section{Data Availability}

The data used to support the findings of this study are included within the article.

\section{Conflicts of Interest}

The authors declare that they have no conflicts of interest regarding the publication of this paper.

\section{Acknowledgments}

This work was supported by Zhejiang Provincial Communication Department [Grant no. 2015J07].

\section{References}

[1] S. Sivaraman and M. M. Trivedi, "Looking at vehicles on the road: a survey of vision-based vehicle detection, tracking, and behavior analysis," IEEE Transactions on Intelligent Transportation Systems, vol. 14, no. 4, pp. 1773-1795, 2013.

[2] B. Yu and Y. Chen, "Driving Comfort Evaluation of Urban Road from Driver's Visual Perception," in Proceedings of the 15th COTA International Conference of Transportation Professionals, pp. 2570-2579, Beijing, China.

[3] T. Deng, K. Yang, Y. Li, and H. Yan, "Where Does the Driver Look? Top-Down-Based Saliency Detection in a Traffic Driving Environment," IEEE Transactions on Intelligent Transportation Systems, vol. 17, no. 7, pp. 2051-2062, 2016.

[4] T. W. Victor, J. L. Harbluk, and J. A. Engström, "Sensitivity of eye-movement measures to in-vehicle task difficulty," Transportation Research Part F: Traffic Psychology and Behaviour, vol. 8, no. 2, pp. 167-190, 2005.

[5] X. Pan, Q. Fang, and H. Jiang, "Driving Visual Demand-based Safety Evaluation of Mountainous Highway Horizontal Curve," Journal of Tongji University. Natural Science, vol. 38, no. 12, pp. 1763-1766, 2010.

[6] W. J. Horrey and C. D. Wickens, "Focal and Ambient Visual Contributions and Driver Visual Scanning in Lane Keeping and Hazard Detection," Proceedings of the Human Factors and Ergonomics Society Annual Meeting, vol. 48, no. 19, pp. 23252329, 2016.

[7] Z. H. Khattak, M. D. Fontaine, and R. A. Boateng, "Evaluating the impact of adaptive signal control technology on driver stress and behavior using real-world experimental data," Transportation Research Part F: Traffic Psychology and Behaviour, vol. 58, pp. 133-144, 2018.

[8] J. Werneke and M. Vollrath, "What does the driver look at? the influence of intersection characteristics on attention allocation and driving behavior," Accident Analysis \& Prevention, vol. 45, pp. 610-619, 2012.

[9] Y. Yun-xing, C. Fang, and Z. Yong-fu, "Research on Visual Behavior Characteristics of Drivers in Downhill Sections of the Mountainous Expressway," Highway, vol. 1, pp. 132-137, 2018.
[10] L. Zhong, X. Zhao, H. Ding et al., "Experimental Research on Safety Impact of Inside Shoulder Width on Drivers without Speed Limitation," Journal of Highway \& Transportation Research Development, 2015.

[11] K. Dixon and L. Brown, "Assessing how drivers of through vehicles react to driveway activity," Transportation Research Record, no. 2404, pp. 77-84, 2014.

[12] O. Oviedo-Trespalacios, M. M. Haque, M. King, and S. Washington, "Effects of road infrastructure and traffic complexity in speed adaptation behaviour of distracted drivers," Accident Analysis \& Prevention, vol. 101, pp. 67-77, 2017.

[13] M. S. Young, J. M. Mahfoud, N. A. Stanton, P. M. Salmon, D. P. Jenkins, and G. H. Walker, "Conflicts of interest: the implications of roadside advertising for driver attention," Transportation Research Part F: Traffic Psychology and Behaviour, vol. 12, no. 5, pp. 381-388, 2009.

[14] S. Bendak and K. Al-Saleh, "The role of roadside advertising signs in distracting drivers," International Journal of Industrial Ergonomics, vol. 40, no. 3, pp. 233-236, 2010.

[15] O. Khatib, J. Warren, V. De Sapio, and L. Sentis, "Human-Like Motion from Physiologically-Based Potential Field," in Building the Information Society, vol. 156 of IFIP International Federation for Information Processing, pp. 747-748, Springer US, Boston, MA, 2004.

[16] Y. Sha and Q. Shi, "Pedestrian Simulation Model based on Discrete Potential Field," Highway Engineering, vol. 34, no. 2, pp. 153-156, 2009.

[17] E. Teh, S. Jamson, and O. Carsten, "Mind the gap: Drivers underestimate the impact of the behaviour of other traffic on their workload," Applied Ergonomics, vol. 67, pp. 125-132, 2018.

[18] T. Sattel, T. Hesse, and C. Sondermann-Wölke, "Experimental study of evasion maneuvers with a potential field-based assistance function for collision avoidance," VDI-Berichte, no. 2009, pp. 475-493, 2008.

[19] H. Wang and T. Wu, "New vector field microcosmic model for traffic flow," China Journal of Highway and Transport, vol. 16, pp. 99-102, 2003 (Chinese).

[20] J. Wang, J. Wu, X. Zheng, D. Ni, and K. Li, “Driving safety field theory modeling and its application in pre-collision warning system," Transportation Research Part C: Emerging Technologies, vol. 72, pp. 306-324, 2016.

[21] J. Wang, J. Wu, and Y. Li, "Concept, Principle and Modeling of Driving Risk Field Based on Driver-vehicle-road Interaction," China Journal of Highway and Transport, vol. 29, pp. 105-114, 2016.

[22] J. Ji, H. Peng, W. Chi et al., "Decision Making for Vehiel Collision Avoidance Based on Dangerous Potential Field," SAEChina Congress \& Exhibition, vol. 2, 2015.

[23] J. Ji, P. Ji, and H. Peng, "Design of 3D Virtual Dangerous Potential Field for Vehicle Active Collision Avoidance," Automotive Engineering Magazine, vol. 38, no. 9, pp. 1065-1071, 2016.

[24] R. V. Ponnaluri and Y. D. Santhi, "Road crash history and risk groups in India: Need for new initiatives and safety policies," Transportation Research Record, no. 2114, pp. 64-71, 2009.

[25] S. Peter, P. Maurer, and R. Stütz, MARVIN-Model for Assessing Risks of Road Infrastructure, Arsenal Research, Vienna, Austria, 2007.

[26] I. Appleton, "Road Infrastructure Safety Assessment," in Proceedings of the 4th IRTAD Conference, Seoul, Korea, 2009.

[27] D. Zhang and X. Hu, "Risk Assessment of Traffic Facility on Freeway Based on a Modified Bayesian Network Model," 
Journal of Transport Information and Safety, vol. 34, no. 5, pp. 102-107, 2016.

[28] P. Gehl, Bayesian Networks for The Multi-Risk Assessment of Road Infrastructure, UCL (University College London), 2017.

[29] A. Weninger-Vycudil, B. Brozek, R. Spielhofer, C. Britton, and M. Oldfield, "Integration of cross asset risk assessment into road infrastructure asset management," in Proceedings of the IABSE Conference, Geneva 2015: Structural Engineering: Providing Solutions to Global Challenges, pp. 1010-1016, Switzerland, September 2015.

[30] J. Bald, K. Stumpf, and T. Wallrabenstein, "Systematic risk analysis for safety assessments of road systems," Journal of the Acoustical Society of America, vol. 123, no. 5, pp. 3466-3466, 2008.

[31] S. SUGIURA, Y. KANAMORI, A. TAKAGI, F. KURAUCHI, and H. MORIMOTO, "Development of integrated asset management technique of road infrastructure based on risk evaluation," Journal of Japan Society of Civil Engineers, Ser. F4 (Construction and Management), vol. 67, no. 4, pp. I_103-I_112, 2011.

[32] M. S. Nemmang, R. Rahman, M. M. Rohani et al., "Analysis of Speeding Behaviour During Approaching the U-Turn Facility Road Segment Based On Driving Simulation Test," Matec Web of Conferences, vol. 103, p. 08008, 2017.

[33] G. K. Kountouriotis and N. Merat, "Leading to distraction: Driver distraction, lead car, and road environment," Accident Analysis \& Prevention, vol. 89, pp. 22-30, 2016.

[34] S. Matena, A. Hegewald, W. Louwerse et al., "Reduction of head-on collisions and run-off-the-road-accident. Report D 3.2 of the RiPCORD-iSEREST project (Road Infrastructure Safety Protection - Core-Research and Development for Road Safety in Europe; Increasing safety and reliability of secondary road)," 2009.

[35] M. Tziotis, V. Pyta, N. Mabbott et al., "Road safety engineering risk assessment part 10: rural run-off-road crashes," Ran Off Road Crashes, 2010.

[36] J. Schorr, S. H. Hamdar, and C. Silverstein, "Measuring the safety impact of road infrastructure systems on driver behavior: Vehicle instrumentation and real world driving experiment," Journal of Intelligent Transportation Systems: Technology, Planning, and Operations, vol. 21, no. 5, pp. 364-374, 2017.

[37] M. Gothié, V. Cerezo, and F. Conche, "Relationship between road infrastructure characteristics and HGV accidents," in Proceedings of the International Conference on Heavy Vehicles, HVTT10: 10th International Symposium on Heavy Vehicle Transportation Technologies, pp. 319-331, 2008.

[38] R. Jobanputra and M. Vanderschuren, "Micro-simulation modelling of the impact of infrastructure provision and vehicle and pedestrian behaviour on road crash risk," in Proceedings of the 3rd International Conference on Road Safety and Simulation, 2011.

[39] B. Scott-Parker, C. Jones, and J. Tucker, "Driver stress in response to infrastructure and other road users: simulator research informing an innovative approach to improving road safety," in Proceedings of the Australasian Road Safety Conference 2016, Canberra, ACT, Australia, 2016.

[40] S. H. Hamdar, L. Qin, and A. Talebpour, "Weather and road geometry impact on longitudinal driving behavior: Exploratory analysis using an empirically supported acceleration modeling framework," Transportation Research Part C: Emerging Technologies, vol. 67, pp. 193-213, 2016.
[41] K. McCann and M. D. Fontaine, "Assessing driver speed choice in fog with the use of visibility data from road weather information systems," Transportation Research Record, vol. 2551, pp. 90-99, 2016.

[42] Yi Li, Yuren Chen, and Fan Wang, "The Impact of Traffic Environmental Vision Pressure on Driver Behaviour," Journal of Advanced Transportation, vol. 2018, Article ID 4941605, 12 pages, 2018. 


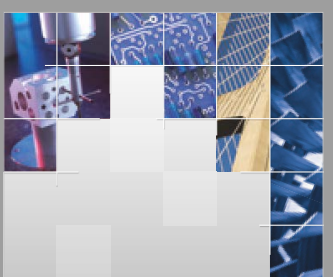

\section{Enfincering}
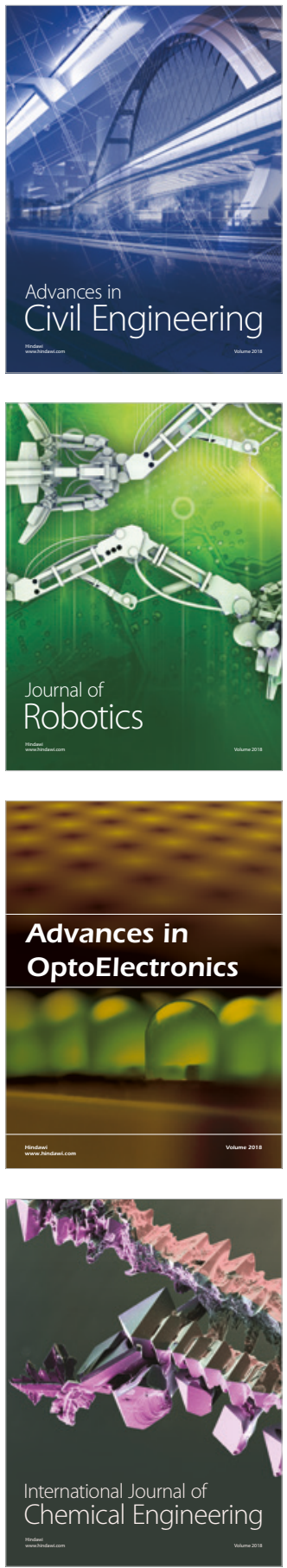

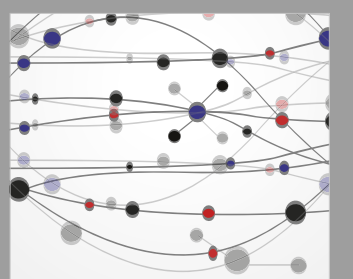

\section{Rotating \\ Machinery}

The Scientific World Journal

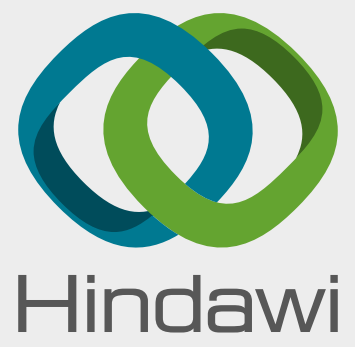

Submit your manuscripts at

www.hindawi.com
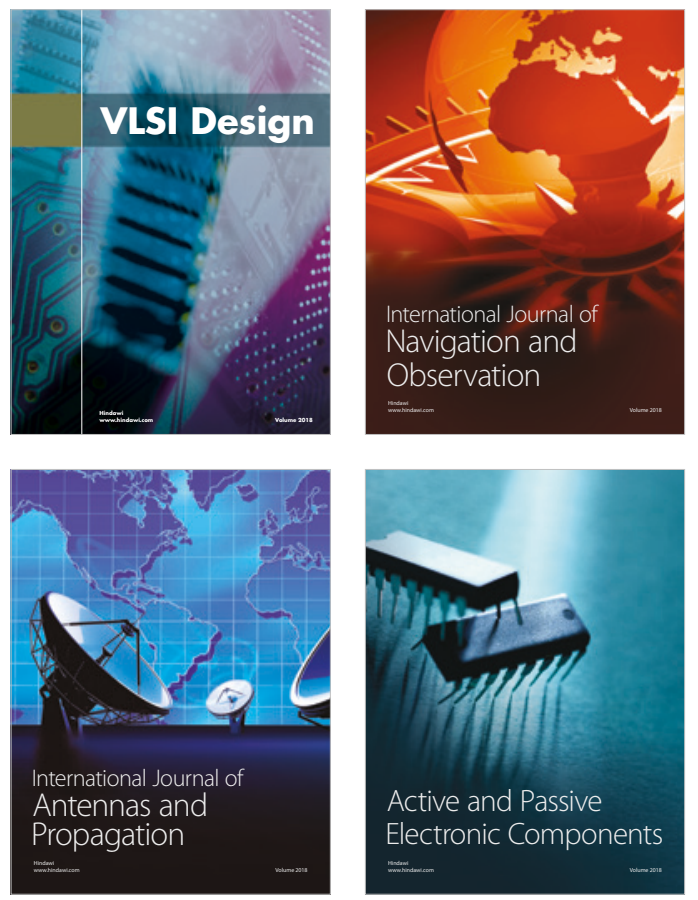
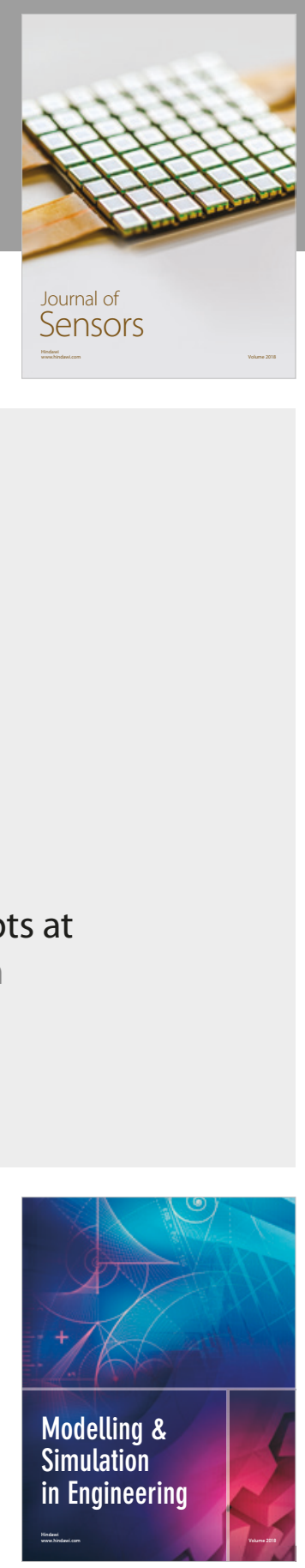

\section{Advances \\ Multimedia}
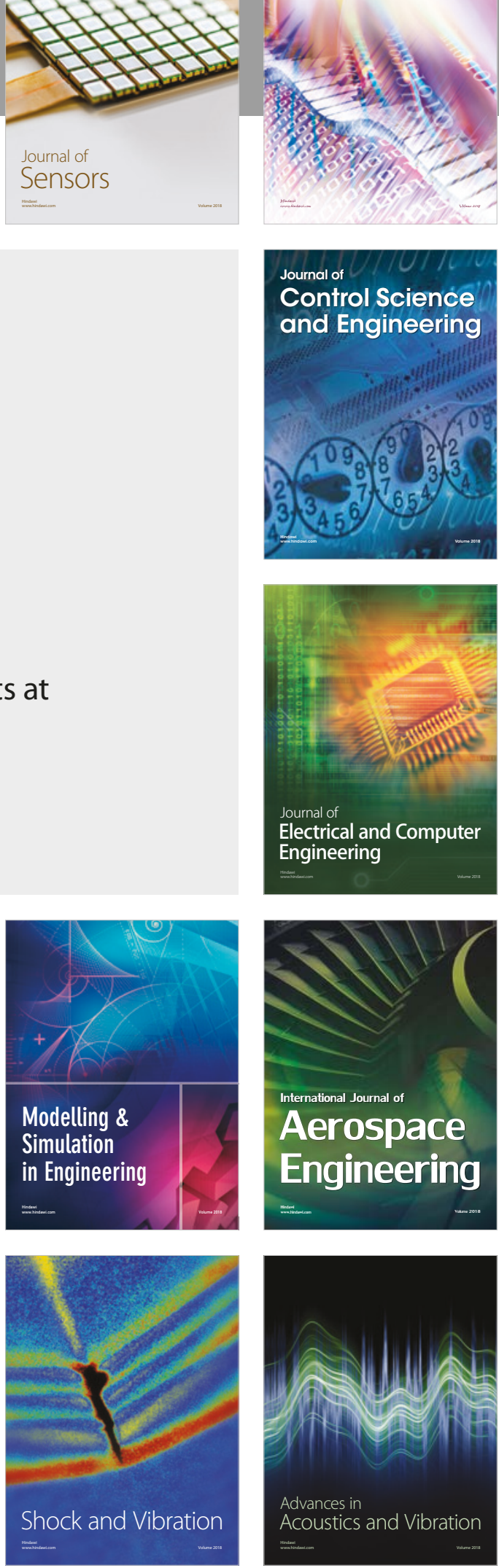